\title{
Orthonormal Bernoulli Polynomials for Solving a Class of Two Dimensional Stochastic Volterra-Fredholm Integral Equations
}

\author{
Ahmad Pourdarvish ${ }^{1} \cdot$ Khosro Sayevand $^{2} \cdot$ Iman Masti $^{2} \cdot$ Sunil Kumar $^{3}$ \\ Accepted: 5 January 2022 / Published online: 22 January 2022 \\ (c) The Author(s), under exclusive licence to Springer Nature India Private Limited 2022
}

\begin{abstract}
Analyzing the mathematical models involving Itô integral, in particular in science and engineering has received much attention, and the reason for this issue is the randomness and lack of access to the exact answer of this type of models. For this purpose, in this paper, the approximate solution of two dimensional (2-D) stochastic Volterra-Fredholm integral equations (SVFIEs) based on the operational matrix method and orthonormal Bernoulli polynomials (OBP) is investigated. Some results and convergence analysis are also presented. Finally, by presenting three examples and reviewing the results and numerical comparisons, we showed that the proposed method has an excellent performance.
\end{abstract}

Keywords Orthonormal Bernoulli polynomials · Stochastic Volterra-Fredholm integral equations · Itô integral · Error analysis · Operational matrix

Mathematics Subject Classification 11B68 - 91G30 - 65C30 - 65R10 · 45B05 - 45D05

\section{Introduction and Basic Definitions}

Mathematical models can greatly assist researchers around the world. Many researchers employed theoretical frameworks and numerical simulations to survey the manner of transmission of different infectious diseases. Recently, Babei et al. [1] examined a stochastic

Khosro Sayevand

ksayehvand@malayeru.ac.ir

Ahmad Pourdarvish

a.pourdarvish@umz.ac.ir

Iman Masti

iman.masti@stu.malayeru.ac.ir

Sunil Kumar

skumar.math@nitjsr.ac.in

1 Department of Statistics, University of Mazandaran, Babolsar, Iran

2 Faculty of Mathematical Sciences, Malayer University, Malayer, Iran

3 Department of Mathematics, National Institute of Technology, Jamshedpur, Jharkhand 831014, India 
model for the transmission of Coronavirus, which is a recent major crisis in many communities. Ganji et al. [2] presented a mathematical model of a brain tumor. This model was an extension of a simple two-dimensional mathematical model of glioma growth and diffusion. In [3] the Klein-Gordon equation (KGE) which has many scientific applications such as quantum field theory, nonlinear optics and solid state physics is investigated. Jafari et al. [4], examined the population dynamics model, including the hunting-hunting problem where the logistics equation, is generalized using the fractional operator.

In many cases, real problems are dependent on some random factors which were ignored due to poor computational power. By increasing the computational power in recent years, these problems are modeled by various types of stochastic equations. Stochastic processes occur in many real issues such as control systems [5], biological population growth [6], biology and medicine [7]. In recent decades, due to the importance of stochastic differential equations (SDE) and stochastic integral equations (SIE) in modeling programs where there is considerable uncertainty, scientists have studied the stochastic process and its applications [8-12].

The 2D Volterra-Fredholm integral equations are an important class of multi dimensional integral equations which arise in various physical and biological models. These equations can be rarely solved exactly and computational complexity of mathematical operations is important obstacle for solving high dimensional stochastic integral equations. Therefore obtaining their numerical solutions have attracted the attention of researchers in numerical analysis branch and some numerical approaches have been presented to solve stochastic integral equations. Such as: operational matrix method based on hat functions [13] and block-pulse [14]. Also, some studies have been performed on different types of stochastic differential integral equations [15-17].

Until now, the number of published papers on numerical solution of multi dimensional stochastic integral equations are very few. Therefore, in this framework this study deals with the numerical solution of 2-D SVFIEs in the following form

$$
\begin{aligned}
z(u, v)= & g(u, v)+\int_{0}^{1} \int_{0}^{1} k_{1}(u, v, e, w) z(e, w) d w d e \\
& +\int_{0}^{u} \int_{0}^{v} k_{2}(u, v, e, w) z(e, w) d w d e \\
& +\int_{0}^{u} \int_{0}^{v} k_{3}(u, v, e, w) z(e, w) d \mathcal{B}(w) d \mathcal{B}(e),
\end{aligned}
$$

where $(u, v) \in[0,1] \times[0,1]$. In this equation, $g(u, v)$ and $k_{i}(u, v, e, w)$ for $i=1,2,3$ are known functions and $z(u, v)$ are unknown functions that we try to determine with our numerical method and $\mathcal{B}=\{\mathcal{B}(t), t \geq 0\}$ is a Brownian process. Also in this equation $\int_{0}^{u} \int_{0}^{v} k_{3}(u, v, e, w) z(e, w) d \mathcal{B}(w) d \mathcal{B}(e)$ is 2-D Itô integral.

This study applies the operational matrix method based on the OBP to obtain the approximate solution of the SVFIEs and the analysis of convergence of the proposed method is discussed.

\section{Itô Integral}

In this section, we briefly introduce the Itô integral and its properties. The reader can refer to references $[8,16,17]$ for more information. 
Definition 1.1 [8,18] For $t \in[0, T], \mathcal{B}(t)$ is called the Brownian motion, if it satisfies the following properties:

i. For $t \geq 0, \mathcal{B}(t)$ is a continuous function of $t$.

ii. For $t>t_{0}, \mathcal{B}(t)-\mathcal{B}\left(t_{0}\right)$, is independent of the past.

iii. $\mathcal{B}(t)-\mathcal{B}\left(t_{0}\right)$ has normal distribution i.e., $E\left(\mathcal{B}(t)-\mathcal{B}\left(t_{0}\right)\right)=0$ and $\operatorname{var}\left(\mathcal{B}(t)-\mathcal{B}\left(t_{0}\right)\right)=$ $t-t_{0}$.

Remark 1.2 By using property (ii), we consider $\mathcal{B}(0)=0$ [16].

Definition 1.3 Assume now that $g \in \Delta(U, V)$ and $\varphi_{m}$ is a sequence of elementary functions which satisfies in the following form

$$
E\left[\int_{U}^{V}\left(g(v, l)-\varphi_{m}(v, l)\right)^{2} d v\right]=0 \text {, as } m \rightarrow \infty,
$$

then, the Itô integral of $g$ [17] is defined as follows:

$$
\int_{U}^{V} g(v, l) d \mathcal{B}_{v}(l)=\lim _{m \rightarrow \infty} \int_{U}^{V} \varphi_{m}(v, l) d \mathcal{B}_{v}(l) .
$$

Property 1.4 One of the valuable properties of Itô integral is as follows [17]:

$$
\int_{0}^{v} g(u) d \mathcal{B}_{u}=g(v) \mathcal{B}_{v}-\int_{0}^{v} \mathcal{B}(u) d g_{u}
$$

\section{2-D Orthonormal Bernoulli Polynomials}

Definition 1.5 The Bernoulli polynomial of order $m$ is defined as follows [19]:

$$
\sum_{i=0}^{m} \frac{(m+1) !}{i !(m+1-i) !} B_{i}(t)=(m+1) t^{m}
$$

One of the features of polynomials in the finite-dimensional, that makes them effective in numerical methods is their orthogonality. But Bernoulli polynomials are not orthogonal, despite their very useful properties [19]. Using Gram-Schmidt orthogonalization process, these polynomials can be orthogonalized as defined below:

Definition 1.6 The orthonormal Bernoulli polynomial of order $m$ is defined as follows:

$$
B_{m}(t)=\sqrt{2 m+1} \sum_{i=0}^{m}(-1)^{i}\left(\begin{array}{c}
m \\
i
\end{array}\right)\left(\begin{array}{c}
2 m-i \\
m-i
\end{array}\right) t^{m-i}, \quad m=0,1,2, \ldots
$$

Therefore, according to the orthogonal property, we will have:

$$
\int_{0}^{1} B_{i}(t) B_{j}(t) d t= \begin{cases}1, & i=j \\ 0, & \text { otherwise. }\end{cases}
$$

In this case, we express a set of OBP as follows:

$$
\mathbb{B}(u)=\left[B_{0}(u), B_{1}(u), \ldots, B_{m}(u)\right]^{T} .
$$


Definition 1.7 2-D orthonormal Bernoulli polynomial of order $m, n$ is defined as follows:

$$
B_{m, n}(u, v)=B_{m}(u) B_{n}(v) \quad m, n=0,1,2, \ldots,
$$

which applies to the following property:

$$
\int_{0}^{1} \int_{0}^{1} B_{m, n}(u, v) B_{p, q}(u, v) d u d v= \begin{cases}1, & m=p, n=q, \\ 0, & \text { otherwise. }\end{cases}
$$

Definition 1.8 The 2-D OBP vector $\mathbb{B}(u, v)$ is defined as:

$$
\begin{aligned}
\mathbb{B}(u, v)= & {\left[B_{0,0}(u, v), B_{0,1}(u, v), \ldots, B_{0, m}(u, v), \ldots, B_{m, 0}(u, v), B_{m, 1}(u, v), \ldots, B_{m, m}\right.} \\
& (u, v)]^{T},
\end{aligned}
$$

or

$$
\mathbb{B}(u, v)=\mathbb{B}(u) \otimes \mathbb{B}(v),
$$

where $\mathbb{B}(u)$ and $\mathbb{B}(v)$ are one-dimensional OBP vector and $\otimes$ is the Kronecker product.

Assume now that $\mathbb{B}(u)=\left[B_{0}(u), B_{1}(u), \ldots, B_{m}(u)\right]^{T}$. Then, by using Eq. (1.6) we obtain

$$
\mathbb{B}(u)=A T_{m}(u),
$$

where

$$
T_{m}(u)=\left[1, u, \ldots, u^{m}\right]^{T},
$$

and $A_{(m+1) \times(m+1)}$ is signified by

$$
A=\left(\begin{array}{cccc}
(-1)^{0}\left(\begin{array}{c}
0 \\
0
\end{array}\right)\left(\begin{array}{c}
0 \\
0
\end{array}\right) & 0 & \ldots & 0 \\
(-1)^{1} \sqrt{3}\left(\begin{array}{c}
1 \\
1
\end{array}\right)\left(\begin{array}{c}
1 \\
0
\end{array}\right) & (-1)^{0} \sqrt{3}\left(\begin{array}{l}
1 \\
0
\end{array}\right)\left(\begin{array}{l}
2 \\
1
\end{array}\right) & \cdots & 0 \\
\vdots & \vdots & \ddots & \vdots \\
(-1)^{m} \sqrt{2 m+1}\left(\begin{array}{c}
m \\
m
\end{array}\right)\left(\begin{array}{c}
m \\
0
\end{array}\right) & (-1)^{m-1} \sqrt{2 m+1}\left(\begin{array}{c}
m \\
m-1
\end{array}\right)\left(\begin{array}{c}
m+1 \\
1
\end{array}\right) & \ldots & (-1)^{0} \sqrt{2 m+1}\left(\begin{array}{c}
m \\
0
\end{array}\right)\left(\begin{array}{c}
2 m \\
m
\end{array}\right)
\end{array}\right) .
$$

Since $\operatorname{det}(A)=|A| \neq 0$, therefore:

$$
T_{m}(u)=A^{-1} \mathbb{B}(u) .
$$

If $\mathbb{B}(u)=A T_{m}(u)$ and $\mathbb{B}(v)=A T_{m}(v)$, then:

$$
\begin{aligned}
\mathbb{B}(u, v) & =\mathbb{B}(u) \otimes \mathbb{B}(v) \\
& =A T_{m}(u) \otimes A T_{m}(v) \\
& =(A \otimes A)\left(T_{m}(u) \otimes T_{m}(v)\right) \\
& =\widehat{A} T_{m}(u, v),
\end{aligned}
$$

where

$$
T_{m}(u, v)=\left[1, v, \ldots, v^{m}, u, u v, \ldots, u v^{m}, \ldots, u^{m}, u^{m} v, \ldots, u^{m} v^{m}\right]^{T} .
$$

We can approximate the any function of two variables in the interval $\Lambda=[0,1] \times[0,1]$ as follows:

$$
f(u, v) \simeq f_{m}(u, v)=\sum_{i=0}^{m} \sum_{j=0}^{m} B_{i}(u) c_{i j} B_{j}(v)=\mathbb{B}^{T}(u) c \mathbb{B}(v),
$$


where $c=\left[c_{i j}\right]_{(m+1) \times(m+1)}$ and

$$
c_{i j}=\int_{0}^{1} \int_{0}^{1} B_{i}(u) f(u, v) B_{j}(v) d v d u, i, j=0,1, \ldots, m .
$$

By doing calculations, it can easily be shown that:

$$
f(u, v) \simeq C^{T}(\mathbb{B}(u) \otimes \mathbb{B}(v)):=C^{T} \mathbb{B}(u, v),
$$

where $C$ is defined as follows:

$$
C=\left[c_{00}, c_{01}, \ldots, c_{0 m}, c_{10}, c_{11}, \ldots, c_{1 m}, \ldots, c_{m 0}, c_{m 1}, \ldots, c_{m m}\right]^{T} .
$$

Similarly, an arbitrary function $k(r, s, e, w)$ is estimated via 2-D OBP as:

$$
k(r, s, e, w) \simeq \mathbb{B}^{T}(r, s) K \mathbb{B}(e, w)=\mathbb{B}^{T}(e, w) K^{T} \mathbb{B}(r, s),
$$

where $K$ is a matrix of order $(m+1)^{2} \times(m+1)^{2}$.

\section{Some Properties of Operational Matrices}

Assume that $\mathbb{B}(u)=\left[B_{0}(u), B_{1}(u), \ldots, B_{m}(u)\right]^{T}$. Then, we conclude that

$$
\int_{0}^{1} \mathbb{B}(u) \mathbb{B}^{T}(u) d u=\int_{0}^{1}\left(\begin{array}{cccc}
B_{0}(u) B_{0}(u) & B_{0}(u) B_{1}(u) & \ldots & B_{0}(u) B_{m}(u) \\
B_{1}(u) B_{0}(u) & B_{1}(u) B_{1}(u) & \ldots & B_{1}(u) B_{m}(u) \\
\vdots & \vdots & \ddots & \vdots \\
B_{m}(u) B_{0}(u) & B_{m}(u) B_{1}(u) & \ldots & B_{m}(u) B_{m}(u)
\end{array}\right) d u
$$

therefore:

$$
\int_{0}^{1} \mathbb{B}(u) \mathbb{B}^{T}(u) d u=I_{(m+1) \times(m+1)} .
$$

By performing calculations from Eqs. (1.12) and (2.2), we deduce that

$$
\int_{0}^{1} \int_{0}^{1} \mathbb{B}(u, v) \mathbb{B}^{T}(u, v) d u d v=I \otimes I=\widehat{I} .
$$

Theorem 2.1 Assume now that $\mathbb{B}(u)$ be the OBP vector. Consequently, the operational matrix of integration has the following form:

$$
\int_{0}^{u} \mathbb{B}(t) d t \simeq P \mathbb{B}(u)
$$

where $P=A M A^{-1}$. Here, $A$ is given in $E q$. (1.15) and $M$ is defined by:

$$
M=\left(\begin{array}{ccccc}
0 & 1 & 0 & \ldots & 0 \\
0 & 0 & \frac{1}{2} & \ldots & 0 \\
\vdots & \vdots & \vdots & \ldots & \vdots \\
0 & 0 & 0 & \ldots & \frac{1}{m} \\
0 & 0 & 0 & \ldots & 0
\end{array}\right) .
$$


Proof By performing calculations, the result can be easily obtained.

Theorem 2.2 The operational matrix of integration based of integer order on the 2-D OBP vector defined in Eq. (1.11) for mixed variables is as follows:

$$
\int_{0}^{u} \int_{0}^{v} \mathbb{B}(s, t) d s d t=\widehat{P} \mathbb{B}(u, v),
$$

where $\widehat{P}=P \otimes P$.

Proof From Eq. (2.7) we have

$$
\begin{aligned}
\int_{0}^{u} \int_{0}^{v} \mathbb{B}(s, t) d s d t & =\int_{0}^{u} \int_{0}^{v}(\mathbb{B}(s) \otimes \mathbb{B}(t)) d s d t \\
& =\left(\int_{0}^{u} \mathbb{B}(s) d s\right) \otimes\left(\int_{0}^{v} \mathbb{B}(t) d t\right) \\
& =(P \mathbb{B}(u)) \otimes(P \mathbb{B}(v)) \\
& =(P \otimes P)(\mathbb{B}(u) \otimes \mathbb{B}(v)) \\
& =\widehat{P} \mathbb{B}(u, v) .
\end{aligned}
$$

Theorem 2.3 Assume now that $\mathbb{B}(v)$ be OBP vector. Consequently, the stochastic operational matrix of integration based on the OBP can be defined in the following form:

$$
\int_{0}^{u} \mathbb{B}(v) d \mathcal{B}(v) \simeq Q \mathbb{B}(u),
$$

where $Q=A E A^{-1}, A$ is given in $E q$. (1.15) and $E$ is defined as:

$$
E=\left(\begin{array}{cccc}
\mathcal{B}(0.5) & 0 & \ldots 0 & 0 \\
0 & \frac{3}{4} \mathcal{B}(0.5)-\frac{1}{2} \mathcal{B}(0.25) \ldots 0 & 0 \\
\vdots & 0 & \ldots 0\left(1-\frac{m}{4}\right) \mathcal{B}(0.5)-\frac{m}{2^{m}} \mathcal{B}(0.25)
\end{array}\right)
$$

Proof We have:

$$
\begin{aligned}
\int_{0}^{u} T_{m}(v) d \mathcal{B}(v)=\left(\begin{array}{c}
\int_{0}^{u} d \mathcal{B}(v) \\
\int_{0}^{u} v d \mathcal{B}(v) \\
\vdots \\
\int_{0}^{u} v^{m} d \mathcal{B}(v)
\end{array}\right) & =\left(\begin{array}{c}
\mathcal{B}(u) \\
u \mathcal{B}(u)-\int_{0}^{u} \mathcal{B}(v) d v \\
\vdots \\
u^{m} \mathcal{B}(u)-\int_{0}^{u} m v^{m-1} \mathcal{B}(v) d v
\end{array}\right) \\
& =\left(\begin{array}{c}
1 \\
u \\
\vdots \\
u^{m}
\end{array}\right) \mathcal{B}(u)-\left(\begin{array}{c}
0 \\
\int_{0}^{u} \mathcal{B}(v) d v \\
\vdots \\
\int_{0}^{u} m v^{m-1} \mathcal{B}(v) d v
\end{array}\right) \\
& :=(\delta)=\left(\delta_{0}, \delta_{1}, \ldots, \delta_{m}\right)^{T},
\end{aligned}
$$


where

$$
\delta_{i}=u^{i} \mathcal{B}(u)-\int_{0}^{u} i v^{i-1} \mathcal{B}(v) d v, i=0,1, \ldots, m .
$$

Now, by approximating the integral in Eq. (2.9) in sense of trapezoidal rule for $0, \frac{u}{2}, u$, with $h=\frac{u}{2}$ one recovers

$$
\delta_{i}=u^{i} \mathcal{B}(u)-\left(\frac{u}{4}\left(0+2 i\left(\frac{u}{2}\right)^{i-1} \mathcal{B}\left(\frac{u}{2}\right)+i u^{i-1} \mathcal{B}(u)\right)\right) .
$$

Therefore:

$$
\delta_{i}=\left[\left(1-\frac{i}{4}\right) \mathcal{B}(u)-\frac{i}{2^{i}} \mathcal{B}\left(\frac{u}{2}\right)\right] u^{i}, i=0,1, \ldots, m .
$$

We approximate $\mathcal{B}(u)$ and $\mathcal{B}\left(\frac{u}{2}\right), 0 \leq v \leq 1$, by $\mathcal{B}(0.5)$ and $\mathcal{B}(0.25)$, respectively. In other words

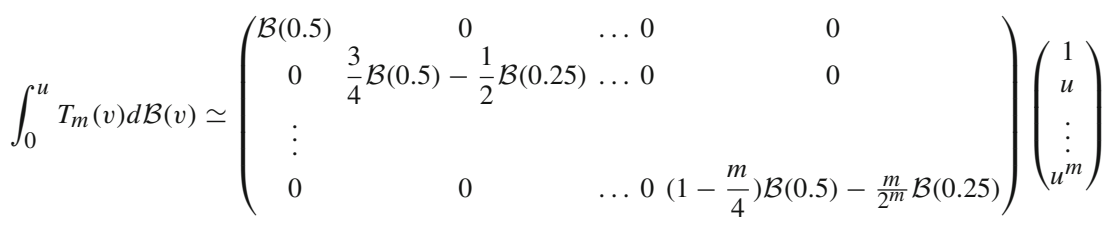

$$
\begin{aligned}
& :=E T_{m}(u) \text {. }
\end{aligned}
$$

By using Eq. (1.13) we can write

$$
\int_{0}^{u} \mathbb{B}(v) d \mathcal{B}(v)=A \int_{0}^{u} T(v) d \mathcal{B}(v) .
$$

Furthermore, by inserting Eq. (2.12) into Eq. (2.13) it results:

$$
\int_{0}^{u} \mathbb{B}(v) d \mathcal{B}(v)=A E T_{m}(u)=A E A^{-1} \mathbb{B}(u):=Q \mathbb{B}(u) .
$$

To obtain $\mathcal{B}(0.5)$ and $\mathcal{B}(0.25)$, in Eq. (2.12) we apply the Definition (1.1), where $\mathcal{B}(t)$ has normal distribution such that:

$$
\mathcal{B}(t)-\mathcal{B}\left(t_{0}\right) \simeq \sqrt{t-t_{0}} N(0,1), \quad t<t_{0} .
$$

Now, assume that $\delta t=\frac{T}{L}$, for some $L \in \mathbb{Z}^{+}$and write $\mathcal{B}_{k}$ for denoting $\mathcal{B}\left(t_{k}\right)$ with $t_{k}=k \delta t$. Remark (1.2) says that $\mathcal{B}(0)=0$ and Definition (1.1) states

$$
\mathcal{B}_{k}=\mathcal{B}_{k-1}+d \mathcal{B}_{k}, k=0,1, \ldots, L,
$$

so that $d \mathcal{B}_{k} ; k=0,1, \ldots, L$ are independent random variable of the form $\sqrt{\delta t} N(0,1)$ [20].

Theorem 2.4 The stochastic operational matrix of integration based of integer order on the 2-D OBP vector defined in Eq. (1.11) is as follows:

$$
\int_{0}^{u} \int_{0}^{v} \mathbb{B}(s, t) d \mathcal{B}(s) d \mathcal{B}(t)=\widehat{Q} \mathbb{B}(u, v),
$$

where $\widehat{Q}=Q \otimes Q$. 
Proof From Eq. (2.14) we have

$$
\begin{aligned}
\int_{0}^{u} \int_{0}^{v} \mathbb{B}(s, t) d \mathcal{B}(s) d \mathcal{B}(t) & =\int_{0}^{u} \int_{0}^{v}(\mathbb{B}(s) \otimes \mathbb{B}(t)) d \mathcal{B}(s) d \mathcal{B}(t) \\
& =\left(\int_{0}^{u} \mathbb{B}(s) d \mathcal{B}(s)\right) \otimes\left(\int_{0}^{v} \mathbb{B}(t) d \mathcal{B}(t)\right) \\
& =(Q \mathbb{B}(u)) \otimes(Q \mathbb{B}(v)) \\
& =(Q \otimes Q)(\mathbb{B}(u) \otimes \mathbb{B}(v)) \\
& =\widehat{Q} \mathbb{B}(u, v) .
\end{aligned}
$$

Theorem 2.5 Assume now that $C_{(m+1)^{2} \times 1}$ is an arbitrary vector. The operational matrix of product $\widehat{C}_{(m+1)^{2} \times(m+1)^{2}}$ using 2-D OBP can be given as follows:

$$
\mathbb{B}(u, v) \mathbb{B}(u, v)^{T} C \simeq \widehat{C} \mathbb{B}(u, v) .
$$

Proof For the first one, from Eq. (1.17) it follows

$$
\begin{aligned}
\mathbb{B}(u, v) \mathbb{B}(u, v)^{T} C= & \widehat{A} T_{m}(u, v) \mathbb{B}(u, v)^{T} C \\
& =\widehat{A}\left(\sum_{i=0}^{m} \sum_{j=0}^{m} c_{i j} B_{i j}(u, v), \sum_{i=0}^{m} \sum_{j=0}^{m} c_{i j} v B_{i j}(u, v), \ldots,\right. \\
& \sum_{i=0}^{m} \sum_{j=0}^{m} c_{i j} v^{m} B_{i j}(u, v), \ldots, \\
& \sum_{i=0}^{m} \sum_{j=0}^{m} c_{i j} u^{m} B_{i j}(u, v), \sum_{i=0}^{m} \sum_{j=0}^{m} c_{i j} u^{m} v B_{i j}(u, v), \ldots, \\
& \left.\sum_{i=0}^{m} \sum_{j=0}^{m} c_{i j} u^{m} v^{m} B_{i j}(u, v)\right)^{T} .
\end{aligned}
$$

Now, we approximate all functions $u^{k} v^{t} B_{i j}(u, v)$ for $k, t=0,1, \ldots, m$ by 2-D OBP:

$$
u^{k} v^{t} B_{i j}(u, v)=\sum_{p=0}^{m} \sum_{q=0}^{m} s_{p q}^{k t, i j} B_{p q}(u, v)=S_{k t, i j}^{T} \mathbb{B}(u, v)
$$

where

$$
S_{k t, i j}=\left[s_{00}^{k t, i j}, s_{01}^{k t, i j}, \ldots, s_{0 m}^{k t, i j}, s_{10}^{k t, i j}, s_{11}^{k t, i j}, \ldots, s_{1 m}^{k t, i j}, \ldots, s_{m 0}^{k t, i j}, s_{m 1}^{k t, i j}, \ldots, s_{m m}^{k t, i j}\right]^{T} .
$$

Furthermore, for $p, q=0,1, \ldots, m$ and $k, t, i, j=0,1, \ldots, m$ we deduce that

$$
s_{p q}^{k t, i j}=\int_{0}^{1} \int_{0}^{1} u^{k} v^{t} B_{i j}(u, v) B_{p q}(u, v) d u d v .
$$


Therefore:

$$
\begin{aligned}
\sum_{i=0}^{m} \sum_{j=0}^{m} c_{i j} u^{k} v^{t} B_{i j}(u, v) & =\sum_{i=0}^{m} \sum_{j=0}^{m} c_{i j}\left(\sum_{p=0}^{m} \sum_{q=0}^{m} s_{p q}^{k t, i j} B_{p q}(u, v)\right) \\
& =\sum_{p=0}^{m} \sum_{q=0}^{m} B_{p q}(u, v)\left(\sum_{i=0}^{m} \sum_{j=0}^{m} c_{i j} s_{p q}^{k t, i j}\right) \\
& =\mathbb{B}(u, v)^{T} \widehat{S}_{k t} C,
\end{aligned}
$$

where $\widehat{S}_{k t}$ is an $(m+1)^{2} \times(m+1)^{2}$ matrix, which is as follows:

$$
\widehat{S}_{k t}=\left(\begin{array}{ccccccccc}
s_{00}^{k t, 00} & s_{00}^{k t, 01} & \ldots & s_{00}^{k t, 0 m}, \ldots & \ldots & s_{00}^{k t, m 0} & s_{00}^{k t, m 1} & \ldots & s_{00}^{k t, m m} \\
s_{01}^{k t, 00} & s_{01}^{k t, 01} & \ldots & s_{01}^{k t, 0 m}, \ldots & \ldots & s_{01}^{k t, m 0} & s_{01}^{k t, m 1} & \ldots & s_{01}^{k t, m m} \\
\vdots & \vdots & \ddots & \vdots & \vdots & \vdots & \vdots & \ddots & \vdots \\
s_{0 m}^{k t, 00} & s_{0 m}^{k t, 01} & \ldots & s_{0 m}^{k t, 0 m}, & \ldots & s_{0 m}^{k t, m 0} & s_{0 m}^{k t, m 1} & \ldots & s_{0 m}^{k t, m m} \\
\vdots & \vdots & \ddots & \vdots & \vdots & \vdots & \vdots & \ddots & \vdots \\
s_{m 0}^{k t, 00} & s_{m 0}^{k t, 01} & \ldots & s_{m 0}^{k t, 0 m}, & \ldots & s_{m 0}^{k t, m 0} & s_{m 0}^{k t, m 1} & \ldots & s_{m 0}^{k t, m m} \\
s_{m 1}^{k t, 00} & s_{m 1}^{k t, 01} & \ldots & s_{m 1}^{k t, 0 m}, & \ldots & s_{m 1}^{k t, m 0} & s_{m 1}^{k t, m 1} & \ldots & s_{m 1}^{k t, m m} \\
\vdots & \vdots & \ddots & \vdots & \vdots & \vdots & \vdots & \ddots & \vdots \\
s_{m m}^{k t, 00} & s_{m m}^{k t, 01} & \ldots & s_{m m}^{k t, 0 m}, \ldots & \ldots & s_{m m}^{k t, m 0} & s_{m m}^{k t, m 1} & \ldots & s_{m m}^{k t, m m}
\end{array}\right) .
$$

Now, we define $E_{k t}=\widehat{S}_{k t} C$ where:

$$
E_{k t}=\left(\begin{array}{c}
\sum_{i=0}^{m} \sum_{j=0}^{m} c_{i j} s_{00}^{k t, i j} \\
\sum_{i=0}^{m} \sum_{j=0}^{m} c_{i j} s_{01}^{k t, i j} \\
\vdots \\
\sum_{i=0}^{m} \sum_{j=0}^{m} c_{i j} s_{0 m}^{k t, i j} \\
\vdots \\
\sum_{i=0}^{m} \sum_{j=0}^{m} c_{i j} s_{m 0}^{k t, i j} \\
\sum_{i=0}^{m} \sum_{j=0}^{m} c_{i j} s_{m 1}^{k t, i j} \\
\vdots \\
\sum_{i=0}^{m} \sum_{j=0}^{m} c_{i j} s_{m m}^{k t, i j}
\end{array}\right) .
$$

Consequently, by defining $\widehat{E}=\left[E_{00}, E_{01}, \ldots, E_{0 m}, E_{10}, E_{11}, \ldots, E_{1 m}, \ldots, E_{m 0}\right.$, $\left.E_{m 1}, \ldots, E_{m m}\right]$, we elicit

$$
\mathbb{B}(u, v) \mathbb{B}(u, v)^{T} C=\widehat{A} \widehat{E} \mathbb{B}(u, v):=\widehat{C} \mathbb{B}(u, v) .
$$




\section{Method of Solution}

Hereunder, we employ the operational matrix method based on 2-D OBP for solving Eq. (1.1). For this purpose we expand $z(u, v), g(v, u)$ and $k_{i}(u, v)$ for $i=1,2,3$ with 2-D OBP so that:

$$
\begin{array}{r}
z(u, v) \simeq z_{m}(u, v)=Z^{T} \mathbb{B}(u, v), \\
g(u, v) \simeq g_{m}(u, v)=G^{T} \mathbb{B}(u, v), \\
k_{i}(u, v, e, w) \simeq \mathbb{B}^{T}(u, v) K_{i} \mathbb{B}(e, w), \quad i=1,2,3,
\end{array}
$$

with $Z, G, \mathbb{B}(u, v), K_{1}, K_{2}$ and $K_{3}$ as defined in the previous section. We will now examine the integrals in Eq. (1.1), respectively. In this way we will have:

$$
\begin{aligned}
\int_{0}^{1} \int_{0}^{1} k_{1}(u, v, e, w) z(e, w) d w d e & =\int_{0}^{1} \int_{0}^{1} \mathbb{B}^{T}(u, v) K_{1} \mathbb{B}(e, w) \mathbb{B}^{T}(e, w) Z d w d e \\
& =\mathbb{B}^{T}(u, v) K_{1} \int_{0}^{1} \int_{0}^{1} \mathbb{B}(e, w) \mathbb{B}^{T}(e, w) d w d e Z \\
& =\mathbb{B}^{T}(u, v) K_{1} \widehat{I Z} .
\end{aligned}
$$

For the second integral in Eq. (1.1) we have:

$$
\begin{aligned}
\int_{0}^{u} \int_{0}^{v} k_{2}(u, v, e, w) z(e, w) d w d e & =\int_{0}^{u} \int_{0}^{v} \mathbb{B}^{T}(u, v) K_{2} \mathbb{B}(e, w) \mathbb{B}^{T}(e, w) Z d w d e \\
& =\mathbb{B}^{T}(u, v) K_{2} \int_{0}^{u} \int_{0}^{v} \mathbb{B}(e, w) \mathbb{B}^{T}(e, w) Z d w d e \\
& =\mathbb{B}^{T}(u, v) K_{2} \int_{0}^{u} \int_{0}^{v} \widehat{Z} \mathbb{B}(e, w) d w d e \\
& =\mathbb{B}^{T}(u, v) K_{2} \widehat{Z} \int_{0}^{u} \int_{0}^{v} \mathbb{B}(e, w) d w d e \\
& =\mathbb{B}^{T}(u, v) K_{2} \widehat{Z} \widehat{P} \mathbb{B}(u, v) .
\end{aligned}
$$

Similarly:

$$
\begin{aligned}
\int_{0}^{u} \int_{0}^{v} k_{3}(u, v, e, w) z(e, w) d \mathcal{B}(w) d \mathcal{B}(e) & \\
& =\int_{0}^{u} \int_{0}^{v} \mathbb{B}^{T}(u, v) K_{3} \mathbb{B}(e, w) \mathbb{B}^{T}(e, w) Z d \mathcal{B}(w) d \mathcal{B}(e) \\
& =\mathbb{B}^{T}(u, v) K_{3} \int_{0}^{u} \int_{0}^{v} \mathbb{B}(e, w) \mathbb{B}^{T}(e, w) Z d \mathcal{B}(w) d \mathcal{B}(e) \\
& =\mathbb{B}^{T}(u, v) K_{3} \int_{0}^{u} \int_{0}^{v} \widehat{Z} \mathbb{B}(e, w) d \mathcal{B}(w) d \mathcal{B}(e) \\
& =\mathbb{B}^{T}(u, v) K_{3} \widehat{Z} \int_{0}^{u} \int_{0}^{v} \mathbb{B}(e, w) d \mathcal{B}(w) d \mathcal{B}(e) \\
& =\mathbb{B}^{T}(u, v) K_{3} \widehat{Z} \widehat{Q} \mathbb{B}(u, v) .
\end{aligned}
$$

By placing Eq. (3.1) to Eq. (3.6) in Eq. (1.1), we have:

$$
\begin{aligned}
\mathbb{B}^{T}(u, v) Z= & \mathbb{B}^{T}(u, v) G+\mathbb{B}^{T}(u, v) K_{1} \widehat{I Z}+\mathbb{B}^{T}(u, v) K_{2} \widehat{Z} \widehat{P} \mathbb{B}(u, v) \\
& +\mathbb{B}^{T}(u, v) K_{3} \widehat{Z} \widehat{Q} \mathbb{B}(u, v) .
\end{aligned}
$$


To calculate the unknown coefficients $Z$ from the above equation, we use suitable collocation points. Thus Eq. (3.7) becomes a linear system with $(m+1)^{2}$ equations and $(m+1)^{2}$ unknowns. After calculating the unknown coefficients with the help of Equation $z(u, v)=\mathbb{B}^{T}(u, v) Z$, we can get the approximate answer of the equation.

\section{Some Results and Convergence Analysis}

Theorem 4.1 Suppose that $z_{m}(u)=Z^{T} \mathbb{B}(u)$ where $Z=\left[z_{0}, z_{1}, \ldots, z_{m}\right]^{T}$, is an approximation of a continuous function $z(u)$ on $[0,1]$ by the 1-D OBP. Then, the coefficients $z_{r}$ for $r=0,1, . ., m$ are bounded as follows:

$$
\left|z_{r}\right| \leq \Xi_{r}
$$

where

$$
\Xi_{r}=\rho \sqrt{2 r+1} \sum_{l=0}^{r}\left(\begin{array}{l}
r \\
l
\end{array}\right)\left(\begin{array}{c}
2 r-l \\
r-l
\end{array}\right),
$$

and $\rho$ is an arbitrary constant such that $|z(u)| \leq \rho$.

Proof Using the OBP, $z(u)$ can be approximated as

$$
z(u)=z_{m}(u)=\sum_{r=0}^{m} z_{r} B_{r}(u),
$$

where $z_{r}$ can be determined by

$$
\begin{aligned}
z_{r} & =\int_{0}^{1} z(u) B_{r}(u) d u=\int_{0}^{1} z(u) \sqrt{2 r+1} \sum_{l=0}^{r}(-1)^{l}\left(\begin{array}{l}
r \\
l
\end{array}\right)\left(\begin{array}{c}
2 r-l \\
r-l
\end{array}\right) u^{r-l} d u \\
& =\sqrt{2 r+1} \sum_{l=0}^{r}(-1)^{l}\left(\begin{array}{l}
r \\
l
\end{array}\right)\left(\begin{array}{c}
2 r-l \\
r-l
\end{array}\right) \int_{0}^{1} z(u) u^{r-l} d u .
\end{aligned}
$$

Since the function $z(u)$ is continuous on $[0,1]$, then

$$
\exists \rho>0, \quad \forall u \in[0,1], \quad|z(u)|<\rho .
$$

Thus, we will have:

$$
\left|z_{r}\right| \leq \rho \sqrt{2 r+1} \sum_{l=0}^{r}(-1)^{l}\left(\begin{array}{l}
r \\
l
\end{array}\right)\left(\begin{array}{c}
2 r-l \\
r-l
\end{array}\right)
$$

Theorem 4.2 Suppose that $z_{m}(u)=Z^{T} \mathbb{B}(u)$ is an approximation of a continuous function $z(u)$ on $[0,1]$ by the 1-D OBP. Then, the error bound is as follows:

$$
\left\|z(u)-z_{m}(u)\right\|_{2} \leq\left(\sum_{i=m+1}^{\infty} \Xi_{i}^{2}\right)^{\frac{1}{2}},
$$

which $\Xi$ is presented in Eq. (4.2). 
Proof Suppose that $z(u)=\sum_{i=0}^{\infty} z_{i} B_{i}(u)$ and $z_{m}(u)=\sum_{i=0}^{m} z_{i} B_{i}(u)$. Therefore:

$$
z(u)-z_{m}(u)=\sum_{i=m+1}^{\infty} z_{i} B_{i}(u) .
$$

Since $\int_{0}^{1} B_{i}(u) B_{j}(u) d u=\delta_{i j}$, then:

$$
\begin{aligned}
\left\|z(u)-z_{m}(u)\right\|_{2}^{2} & =\int_{0}^{1}\left|z(u)-z_{m}(u)\right|^{2} d u \\
& =\int_{0}^{1}\left|\sum_{i=m+1}^{\infty} z_{i} B_{i}(u)\right|^{2} d u \\
& =\sum_{i=m+1}^{\infty} z_{i}^{2} \\
& \leq \sum_{i=m+1}^{\infty} \Xi_{i}^{2} .
\end{aligned}
$$

Theorem 4.3 Suppose $f_{m}(u, v)=Z^{T} \mathbb{B}(u, v)$ is the best approximation for the function $f(u, v)$ by the 2-D OBP. In this case, we have

$$
\left\|f(u, v)-f_{m}(u, v)\right\|_{2}=\mathbb{O}\left(\frac{1}{(m+1) ! 2^{2 m+1}}\right),
$$

which means that if $m \longrightarrow \infty$, then $f_{m}(u, v) \longrightarrow f(u, v)$. Here, $\mathbb{O}$ is a big-(O) notation.

Proof See [21].

Note 4.4 By an argument similar to Theorem (4.3), we have:

$$
\left\|f(u, v)-f_{m}(u, v)\right\|_{2} \leq \frac{\rho}{(m+1) ! 2^{2 m+1}} .
$$

Theorem 4.5 Suppose $u \in[0, \infty)$. In this case, we have

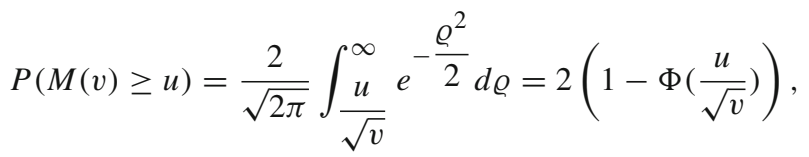

where $M(v)=\sup _{0 \leq r<v} \mathcal{B}(r), P$ is a probability function and $\Phi$ is cumulative standard normal distribution function.

Proof See [22].

Theorem 4.6 Let $z(u, v)$ and $z_{m}(u, v)$ are the exact and 2-D OBP approximate solution of Eq. (1.1). Also $\operatorname{let}_{m}(u, v)$ and $k_{i, m}(u, v, e, w)$ are the 2-D OBP of $g(u, v)$ and $k_{i}(u, v, e, w)$ for $i=1,2,3$, respectively. Also assume that:

(i) $\|z\|_{2} \leq \alpha$,

(ii) $\left\|k_{p}\right\|_{2} \leq \beta_{i}$ for $i=1,2,3$,

(iii) $\mathcal{B}(u, v)=\sup _{u \in[0,1]} \mathcal{B}(u) \sup _{v \in[0,1]} \mathcal{B}(v)$. 
Then, we have:

$$
\begin{aligned}
& \left\|z-z_{m}\right\|_{2} \\
& \leq \frac{\frac{\rho}{(m+1) ! 2^{2 m+1}}(1+2 \alpha+\alpha \mathcal{B}(u, v))}{1-\left(\left(\beta_{1}+\frac{\rho}{(m+1) ! 2^{2 m+1}}\right)+\left(\beta_{2}+\frac{\rho}{(m+1) ! 2^{2 m+1}}\right)+\mathcal{B}(u, v)\left(\beta_{3}+\frac{\rho}{(m+1) ! 2^{2 m+1}}\right)\right)} .
\end{aligned}
$$

Proof We have

$$
\begin{aligned}
z(u, v)= & g(u, v)+\int_{0}^{1} \int_{0}^{1} k_{1}(u, v, e, w) z(e, w) d w d e \\
& +\int_{0}^{u} \int_{0}^{v} k_{2}(u, v, e, w) z(e, w) d w d e \\
& +\int_{0}^{u} \int_{0}^{v} k_{3}(u, v, e, w) z(e, w) d \mathcal{B}(w) d \mathcal{B}(e) .
\end{aligned}
$$

The approximate equation of Eq. (4.12) is:

$$
\begin{aligned}
z_{m}(u, v)= & g_{m}(u, v)+\int_{0}^{1} \int_{0}^{1} k_{1 m}(u, v, e, w) z_{m}(e, w) d w d e \\
& +\int_{0}^{u} \int_{0}^{v} k_{2 m}(u, v, e, w) z_{m}(e, w) d w d e \\
& +\int_{0}^{u} \int_{0}^{v} k_{3 m}(u, v, e, w) z_{m}(e, w) d \mathcal{B}(w) d \mathcal{B}(e) .
\end{aligned}
$$

Therefore, from Eqs. (4.12) and (4.13) we have:

$$
\begin{aligned}
z(u, v)-z_{m}(u, v)= & g(u, v)-g_{m}(u, v) \\
& +\int_{0}^{1} \int_{0}^{1} k_{1}(u, v, e, w) z(e, w)-k_{1 m}(u, v, e, w) z_{m}(e, w) d w d e \\
& +\int_{0}^{u} \int_{0}^{v} k_{2}(u, v, e, w) z(e, w)-k_{2 m}(u, v, e, w) z_{m}(e, w) d w d e \\
& +\int_{0}^{u} \int_{0}^{v} k_{3}(u, v, e, w) z(e, w) \\
& -k_{3 m}(u, v, e, w) z_{m}(e, w) d \mathcal{B}(w) d \mathcal{B}(e) .
\end{aligned}
$$

We now estimate Eq. (4.14) by $2 \mathrm{D}$-integrals mean value theorem for every $(u, v) \in[0,1]^{2}$ and $(u, v, e, w) \in[0,1]^{4}$. Therefore:

$$
\begin{aligned}
\left\|z-z_{m}\right\|_{2}= & \left\|g-g_{m}\right\|_{2}+\left\|k_{1} z-k_{1 m} z_{m}\right\|_{2}+u v\left\|k_{2} z-k_{2 m} z_{m}\right\|_{2} \\
& +\mathcal{B}(u) \mathcal{B}(v)\left\|k_{3} z-k_{3 m} z_{m}\right\|_{2} .
\end{aligned}
$$

On the other hand, for $i=1,2,3$

$$
\left\|k_{i} z-k_{i m} z_{m}\right\|_{2} \leq\left\|k_{i}\right\|_{2}\left\|z-z_{m}\right\|_{2}+\left\|k_{i}-k_{i m}\right\|_{2}\left(\left\|z-z_{m}\right\|_{2}+\|z\|_{2}\right) .
$$


By assumptions ( $i$ ) and (ii) for $i=1,2,3$ and Note (4.4) we obtain:

$$
\begin{aligned}
\left\|k_{i} z-k_{i m} z_{m}\right\|_{2} & \leq\left\|k_{i}\right\|_{2}\left\|z-z_{m}\right\|_{2}+\left\|k_{i}-k_{i m}\right\|_{2}\left(\left\|z-z_{m}\right\|_{2}+\|z\|_{2}\right) \\
& \leq \beta_{i}\left\|z-z_{m}\right\|_{2}+\frac{\rho}{(m+1) ! 2^{2 m+1}}\left(\left\|z-z_{m}\right\|_{2}+\alpha\right) \\
& =\left(\beta_{i}+\frac{\rho}{(m+1) ! 2^{2 m+1}}\right)\left\|z-z_{m}\right\|_{2}+\frac{\alpha \cdot \rho}{(m+1) ! 2^{2 m+1}} .
\end{aligned}
$$

Substituting Eq. (4.17) in Eq. (4.15) and using Note (4.4) implies that:

$$
\begin{aligned}
\left\|z-z_{m}\right\|_{2} \leq & \frac{\rho}{(m+1) ! 2^{2 m+1}}+\left[\left(\beta_{1}+\frac{\rho}{(m+1) ! 2^{2 m+1}}\right)\left\|z-z_{m}\right\|_{2}+\frac{\alpha \cdot \rho}{(m+1) ! 2^{2 m+1}}\right] \\
& +u v\left[\left(\beta_{2}+\frac{\rho}{(m+1) ! 2^{2 m+1}}\right)\left\|z-z_{m}\right\|_{2}+\frac{\alpha \cdot \rho}{(m+1) ! 2^{2 m+1}}\right] \\
& +\mathcal{B}(u) \mathcal{B}(v)\left[\left(\beta_{3}+\frac{\rho}{(m+1) ! 2^{2 m+1}}\right)\left\|z-z_{m}\right\|_{2}+\frac{\alpha \cdot \rho}{(m+1) ! 2^{2 m+1}}\right] .
\end{aligned}
$$

Therefore, we deduce that

$$
\begin{aligned}
\left\|z-z_{m}\right\|_{2} \leq & \frac{\rho}{(m+1) ! 2^{2 m+1}} \\
& +\left[\left(\beta_{1}+\frac{\rho}{(m+1) ! 2^{2 m+1}}\right) \sup _{e \leq u, w \leq v}\left\|z(e, w)-z(e, w)_{m}\right\|_{2}\right. \\
& \left.+\frac{\alpha \cdot \rho}{(m+1) ! 2^{2 m+1}}\right]+1 \times 1 \times\left[\left(\beta_{2}+\frac{\rho}{(m+1) ! 2^{2 m+1}}\right) \sup _{e \leq u, w \leq v} \| z(e, w)\right. \\
& \left.-z(e, w)_{m} \|_{2}+\frac{\alpha \cdot \rho}{(m+1) ! 2^{2 m+1}}\right] \\
& +\mathcal{B}(u, v)\left[\left(\beta_{3}+\frac{\rho}{(m+1) ! 2^{2 m+1}}\right) \sup _{e \leq u, w \leq v}\left\|z(e, w)-z(e, w)_{m}\right\|_{2}\right. \\
& \left.+\frac{\alpha \cdot \rho}{(m+1) ! 2^{2 m+1}}\right] .
\end{aligned}
$$

So:

$$
\left\|z-z_{m}\right\|_{2} \leq \frac{\frac{\rho}{(m+1) ! 2^{2 m+1}}(1+2 \alpha+\alpha \mathcal{B}(u, v))}{1-\left(\left(\beta_{1}+\frac{\rho}{(m+1) ! 2^{2 m+1}}\right)+\left(\beta_{2}+\frac{\rho}{(m+1) ! 2^{2 m+1}}\right)+\mathcal{B}(u, v)\left(\beta_{3}+\frac{\rho}{(m+1) ! 2^{2 m+1}}\right)\right)} .
$$

Note 4.7 By an argument similar to Theorem (4.6), we have:

$$
\left\|z(u, v)-z_{m}(u, v)\right\|_{2}=\mathbb{O}\left(\frac{1}{(m+1) ! 2^{2 m+1}}\right) .
$$

\section{Numerical Feedbacks}

In this section, we show the efficiency and accuracy of the suggested method by giving three examples. All the numerical calculations are performed on an Intel Core i7 laptop by running some programming codes written in MATLAB R2016b. 
Table 1 Absolute error of Example 5.1 for $m=2$, and $m=3$

\begin{tabular}{lll}
\hline$(\mathrm{u}, \mathrm{v})$ & $m=2$ & $m=3$ \\
\hline$(0,0)$ & $4.3509 \times 10^{-12}$ & $1.3028 \times 10^{-13}$ \\
$(0.1,0.1)$ & $1.0652 \times 10^{-12}$ & $2.4236 \times 10^{-13}$ \\
$(0.2,0.2)$ & $3.7256 \times 10^{-11}$ & $1.9625 \times 10^{-11}$ \\
$(0.3,0.3)$ & $2.5477 \times 10^{-11}$ & $1.2239 \times 10^{-12}$ \\
$(0.4,0.4)$ & $2.2593 \times 10^{-10}$ & $1.1144 \times 10^{-11}$ \\
$(0.5,0.5)$ & $3.3341 \times 10^{-10}$ & $1.5870 \times 10^{-12}$ \\
$(0.6,0.6)$ & $1.4257 \times 10^{-11}$ & $1.6791 \times 10^{-13}$ \\
$(0.7,0.7)$ & $2.3569 \times 10^{-11}$ & $2.9614 \times 10^{-12}$ \\
$(0.8,0.8)$ & $2.2910 \times 10^{-11}$ & $4.0209 \times 10^{-12}$ \\
$(0.9,0.9)$ & $1.8456 \times 10^{-10}$ & $3.4962 \times 10^{-12}$ \\
$(1,1)$ & $1.2958 \times 10^{-9}$ & $5.7024 \times 10^{-10}$ \\
\hline
\end{tabular}

Table 2 Comparing maximum absolute error of Example 5.1

\begin{tabular}{|c|c|c|c|}
\hline \multicolumn{4}{|c|}{ Maximum error in $(0.1,0.2)$} \\
\hline $\mathrm{m}$ & {$[14]$} & [13] & Our method \\
\hline 2 & 0.0478382 & $0.117 \times 10^{-11}$ & $0.2796 \times 10^{-12}$ \\
\hline 3 & 0.0072947 & - & $0.1285 \times 10^{-13}$ \\
\hline 4 & 0.0033901 & $0.222 \times 10^{-14}$ & $0.5035 \times 10^{-16}$ \\
\hline & \multicolumn{3}{|c|}{ Maximum error in $(0.3,0.7)$} \\
\hline$\overline{\mathrm{m}}$ & {$[14]$} & [13] & Our method \\
\hline 2 & 0.0351850 & $0.149 \times 10^{-12}$ & $0.3902 \times 10^{-13}$ \\
\hline 3 & 0.0276713 & - & $0.6834 \times 10^{-14}$ \\
\hline 4 & 0.0123877 & $0.244 \times 10^{-14}$ & $0.1295 \times 10^{-16}$ \\
\hline
\end{tabular}

Example 5.1 Assuming $k_{1}(u, v, e, w)=u, k_{2}(u, v, e, w)=u$, and $k_{3}(u, v, e, w)=u+v$ in Eq. (1.1), consider the SVFIE that was studied by $[13,14]$ :

$$
\begin{aligned}
z(u, v)= & g(u, v)+\int_{0}^{1} \int_{0}^{1} u z(e, w) d w d e+\int_{0}^{u} \int_{0}^{v} u z(e, w) d w d e \\
& +\int_{0}^{u} \int_{0}^{v}(u+v) z(e, w) d \mathcal{B}(w) d \mathcal{B}(e) .
\end{aligned}
$$

In this case, assuming $g(u, v)=1-u-u^{2} v-(u+v) \mathcal{B}(u) \mathcal{B}(v)$, the exact solution of the equation is $z(u, v)=1$.

In Table 1, the values of absolute error at different points for $m=2$ and $m=3$ are reported which indicates the accuracy of the suggested scheme. Table 2, provides a comparison of maximum absolute error for different values of $m$ in points $(0.1,0.2)$ and $(0.3,0.7)$ between the presented scheme and previously published algorithms by Fallahpour et al. [14].

Also, absolute error for $m=2$ and $m=3$ are plotted in Figs. 1 and 2 , respectively. By examining the absolute error presented in Figs. 1 and 2 for different values of $m$, we will find that by increasing $m$, the error decreases substantially. 


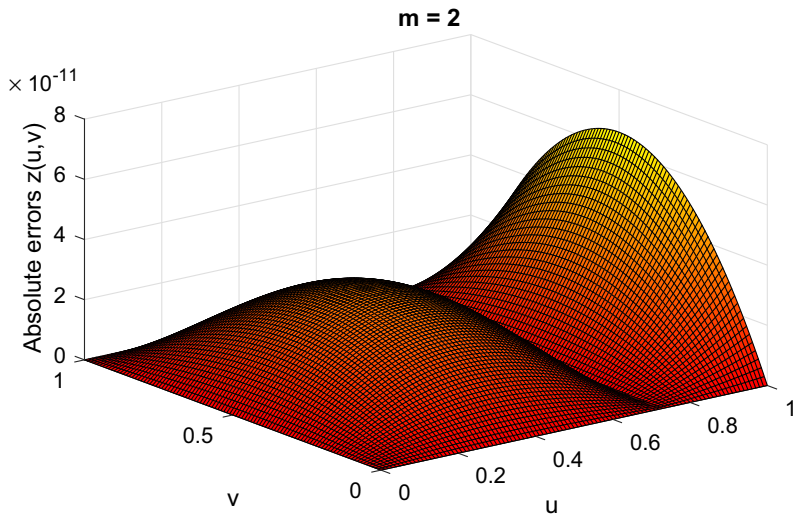

Fig. 1 The absolute errors with $m=2$ for Example 5.1

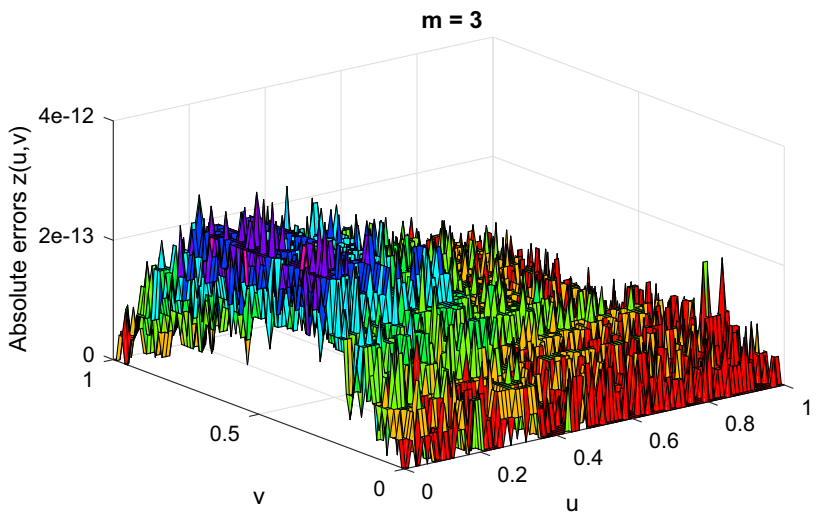

Fig. 2 The absolute errors with $m=3$ for Example 5.1

Example 5.2 Consider the SVFIE that was studied by [14]:

$$
\begin{aligned}
z(u, v)= & g(u, v)+\int_{0}^{1} \int_{0}^{1}(u+v+e+w) z(e, w) d w d e \\
& +\int_{0}^{u} \int_{0}^{v}(u+v+e+w) z(e, w) d w d e \\
& +\int_{0}^{u} \int_{0}^{v}(\text { uvew }) z(e, w) d \mathcal{B}(w) d \mathcal{B}(e),
\end{aligned}
$$

where

$$
g(u, v)=\frac{-7}{6}-\frac{1}{6} u v\left(5 u^{2}+9 u v+5 v^{2}\right)-2 u v\left(u^{2} \mathcal{B}(u)-2 \int_{0}^{u} \mathcal{B}(s) d s\right)\left(v \mathcal{B}(v)-\int_{0}^{v} \mathcal{B}(t) d t\right) .
$$

The exact solution of (5.2) is $z(u, v)=u+v$.

In Table 3, the values of absolute error at different points for $m=2$ and $m=3$ are reported which indicates the accuracy of the suggested scheme. Table 4, provides a comparison of 
Table 3 Absolute error of Example 5.2 for $m=2$, and $m=3$

\begin{tabular}{lll}
\hline$(\mathrm{u}, \mathrm{v})$ & $m=2$ & $m=3$ \\
\hline$(0,0)$ & $3.2398 \times 10^{-5}$ & $0.7164 \times 10^{-9}$ \\
$(0.1,0.1)$ & $1.6148 \times 10^{-5}$ & $3.6453 \times 10^{-8}$ \\
$(0.2,0.2)$ & $2.4195 \times 10^{-4}$ & $1.9021 \times 10^{-8}$ \\
$(0.3,0.3)$ & $2.936 \times 10^{-5}$ & $1.2293 \times 10^{-7}$ \\
$(0.4,0.4)$ & $1.2864 \times 10^{-4}$ & $2.5051 \times 10^{-8}$ \\
$(0.5,0.5)$ & $2.3285 \times 10^{-4}$ & $2.7438 \times 10^{-7}$ \\
$(0.6,0.6)$ & $1.7705 \times 10^{-4}$ & $2.6781 \times 10^{-7}$ \\
$(0.7,0.7)$ & $3.2308 \times 10^{-4}$ & $2.9154 \times 10^{-8}$ \\
$(0.8,0.8)$ & $6.1029 \times 10^{-5}$ & $4.9020 \times 10^{-8}$ \\
$(0.9,0.9)$ & $4.6548 \times 10^{-5}$ & $4.4286 \times 10^{-8}$ \\
$(1,1)$ & $4.5927 \times 10^{-4}$ & $5.3481 \times 10^{-7}$ \\
\hline
\end{tabular}

Table 4 Comparing maximum absolute error of Example 5.2

\begin{tabular}{|c|c|c|}
\hline \multirow[t]{2}{*}{$\mathrm{m}$} & \multicolumn{2}{|l|}{ Maximum error in $(0.1,0.2)$} \\
\hline & {$[14]$} & Our method \\
\hline 2 & 0.1737880 & $1.5267 \times 10^{-4}$ \\
\hline 3 & 0.0105896 & $2.9784 \times 10^{-7}$ \\
\hline 4 & 0.0665286 & $3.7765 \times 10^{-9}$ \\
\hline \multirow[t]{2}{*}{$\mathrm{m}$} & \multicolumn{2}{|l|}{ Maximum error in $(0,0.6)$} \\
\hline & {$[14]$} & Our method \\
\hline 2 & 0.3847700 & $3.4368 \times 10^{-4}$ \\
\hline 3 & 0.0566236 & $5.9157 \times 10^{-8}$ \\
\hline 4 & 0.1313150 & $2.0371 \times 10^{-8}$ \\
\hline
\end{tabular}

maximum absolute error for different values of $m$ in points $(0.1,0.2)$ and $(0,0.6)$ between the presented scheme and previously published algorithms by Fallahpour et al. [14].

Also, absolute error for $m=2$ and $m=3$ are plotted in Figs. 3 and 4, respectively. Three dimensional graphs of the exact and approximation solution is shown in Fig. 5. It can easily be seen from the figures that as the number of $m$ increases the approximate solution converges towards the exact solution. Figure 6 shows the approximate solution and the exact solution at the top and the absolute error at the bottom, for $v=0.5$ and $m=3$.

Example 5.3 Consider the SVFIE that was studied by [14]:

$$
\begin{aligned}
z(u, v)= & g(u, v)+\int_{0}^{1} \int_{0}^{1}(u e) \sin (w+v) z(e, w) d w d e+\int_{0}^{u} \int_{0}^{v}(u e) \sin (w+v) z(e, w) d w d e \\
& +\int_{0}^{u} \int_{0}^{v}(e+w) \cos (u v) z(e, w) d \mathcal{B}(w) d \mathcal{B}(e),
\end{aligned}
$$




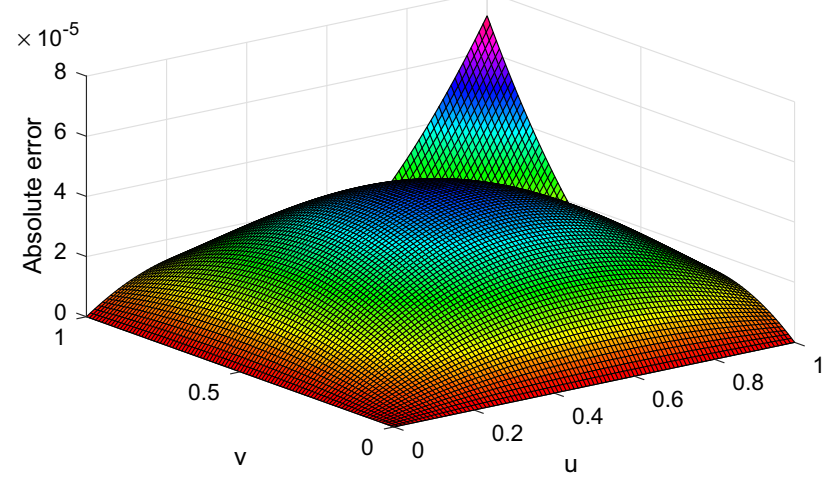

Fig. 3 The absolute errors with $m=2$ for Example 5.2

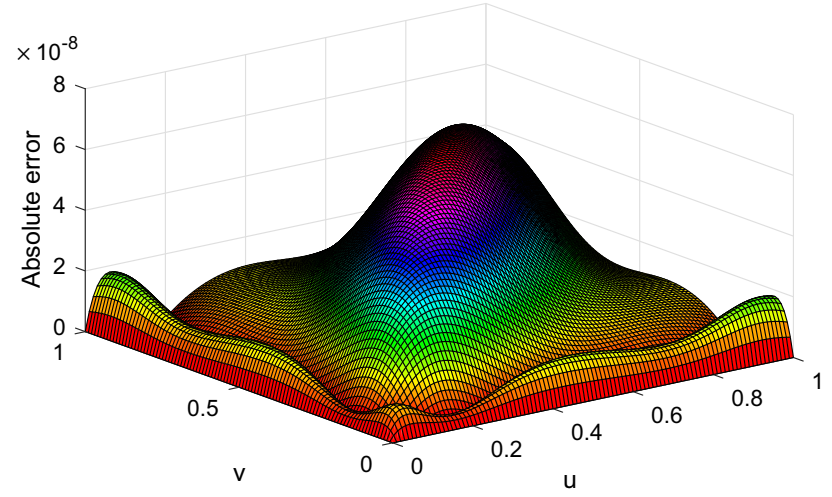

Fig. 4 The absolute errors with $m=3$ for Example 5.2

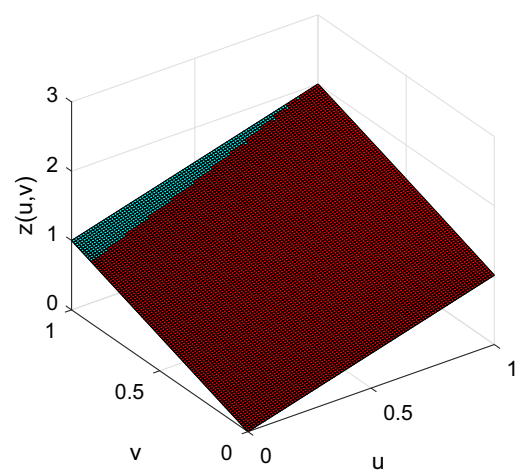

approximate solution exact solution

Fig. 5 The absolute errors with $m=3$ for Example 5.2 

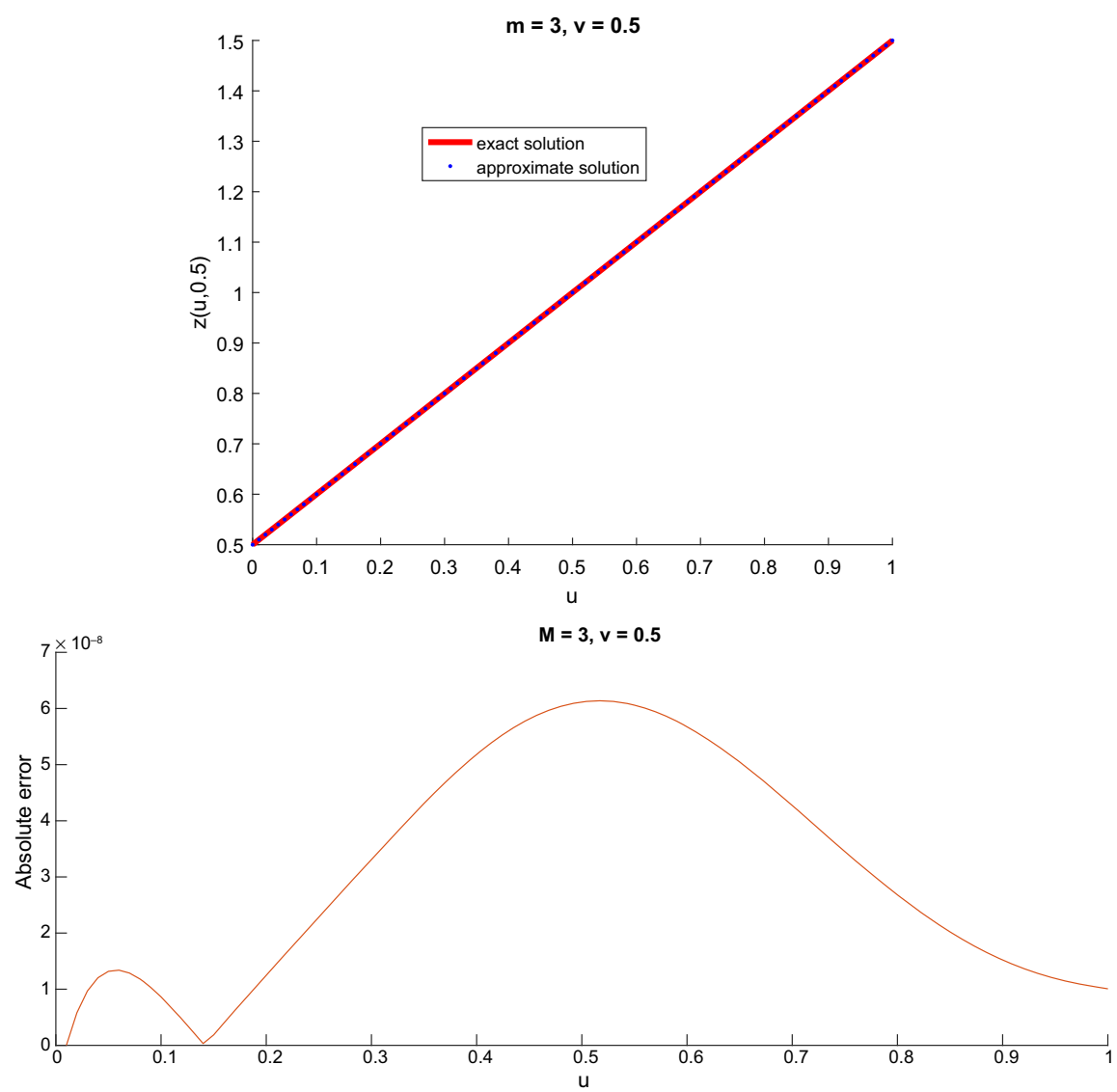

Fig. 6 The approximate solution and the exact solution and absolute errors of Example 5.2 for $v=0.5$ and $m=3$

where

$$
\begin{aligned}
g(u, v)= & u v+\frac{1}{3} u(\cos (1+v)+\sin (v)-\sin (1+v)) \\
& +\frac{1}{3} u^{4}(v \cos (2 v)+\sin (v)-\sin (2 v)) \\
& -\cos (u v)\left(u^{2} \mathcal{B}(u)-2 \int_{0}^{u} e \mathcal{B}(e) d e\right)\left(v \mathcal{B}(v)-\int_{0}^{v} \mathcal{B}(e) d e\right) \\
& -\cos (u v)\left(v^{2} \mathcal{B}(v)-2 \int_{0}^{v} e \mathcal{B}(e) d e\right)\left(u \mathcal{B}(u)-\int_{0}^{u} \mathcal{B}(e) d e\right) .
\end{aligned}
$$

The exact solution of (5.4) is $z(u, v)=u v$.

In Table 5, the values of absolute error at different points for $m=2$ and $m=3$ are reported which indicates the accuracy of the suggested scheme. Table 6 , provides a comparison of maximum absolute error for different values of $m$ in points $(0.6,0.8)$ and $(0.4,0.9)$ between the presented scheme and previously published algorithms in $[13,14]$. 
31 Page 20 of 24

Int. J. Appl. Comput. Math (2022) 8:31

Table 5 Absolute error of Example 5.3 for $m=2$, and $m=3$
Table 6 Comparing maximum absolute error of Example 5.3

\begin{tabular}{lll}
\hline$(\mathrm{u}, \mathrm{v})$ & $m=2$ & $m=3$ \\
\hline$(0,0)$ & $1.294 \times 10^{-5}$ & $0.617 \times 10^{-7}$ \\
$(0.1,0.1)$ & $1.469 \times 10^{-4}$ & $4.806 \times 10^{-6}$ \\
$(0.2,0.2)$ & $6.635 \times 10^{-4}$ & $1.436 \times 10^{-6}$ \\
$(0.3,0.3)$ & $9.891 \times 10^{-4}$ & $1.292 \times 10^{-6}$ \\
$(0.4,0.4)$ & $1.918 \times 10^{-3}$ & $2.970 \times 10^{-6}$ \\
$(0.5,0.5)$ & $2.056 \times 10^{-3}$ & $9.892 \times 10^{-6}$ \\
$(0.6,0.6)$ & $2.870 \times 10^{-3}$ & $1.523 \times 10^{-5}$ \\
$(0.7,0.7)$ & $2.106 \times 10^{-3}$ & $1.119 \times 10^{-5}$ \\
$(0.8,0.8)$ & $1.875 \times 10^{-3}$ & $5.973 \times 10^{-6}$ \\
$(0.9,0.9)$ & $3.042 \times 10^{-5}$ & $2.807 \times 10^{-6}$ \\
$(1,1)$ & $1.597 \times 10^{-3}$ & $4.742 \times 10^{-6}$ \\
\hline
\end{tabular}

\begin{tabular}{lll}
\hline $\mathrm{m}$ & Maximum error in $(0.6,0.8)$ & \\
\cline { 2 - 3 } & {$[14]$} & Our method \\
\hline 2 & 0.7683170 & $6.537 \times 10^{-5}$ \\
4 & 0.156307 & $5.668 \times 10^{-8}$ \\
\hline $\mathrm{m}$ & Maximum error in $(0.4,0.9)$ & \\
\hline & {$[14]$} & Our method \\
\hline 2 & 0.303459 & $8.723 \times 10^{-5}$ \\
4 & 0.179375 & $7.132 \times 10^{-8}$ \\
\hline
\end{tabular}

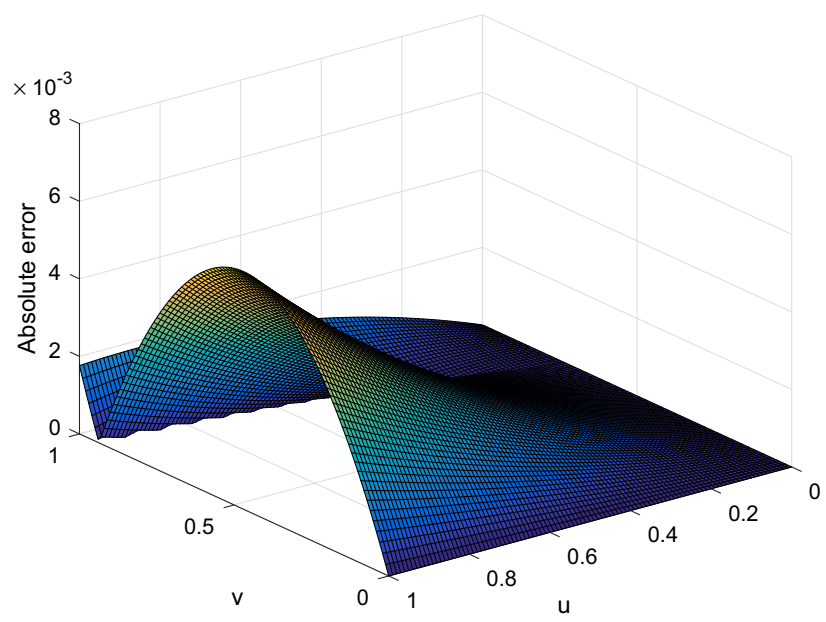

Fig. 7 The absolute errors with $m=2$ for Example 5.3

Springer 


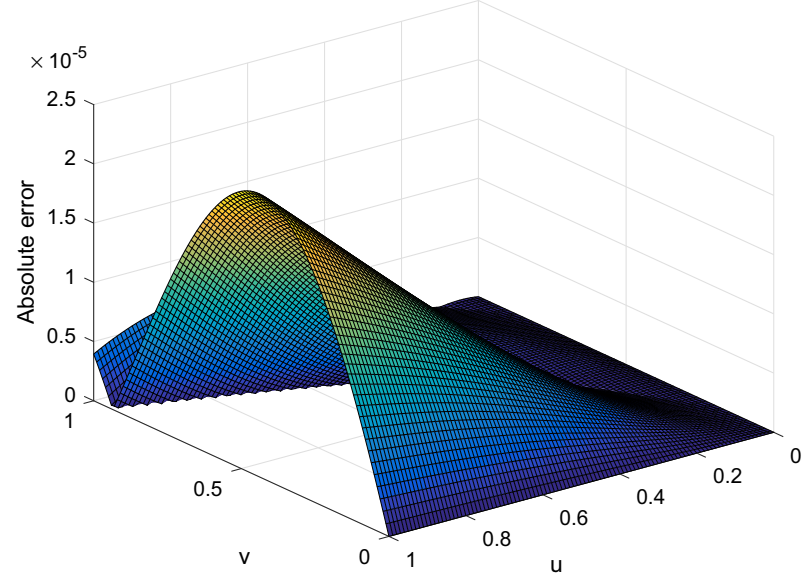

Fig. 8 The absolute errors with $m=3$ for Example 5.3

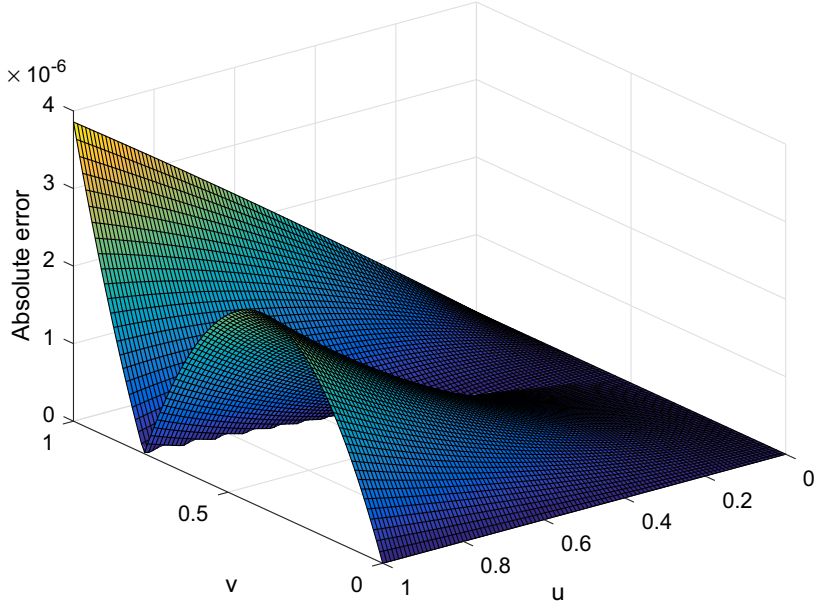

Fig. 9 The absolute errors with $m=4$ for Example 5.3

Also, absolute error for $m=2, m=3$ and $m=4$ are plotted in Figs. 7, 8 and 9, respectively. Figure 10 shows the approximate solution and the exact solution for $v=0.5$ and $m=3$ at the top and the 3-D graphs of the approximation solution at the bottom.

\section{Conclusion}

Obtaining approximate answers for Itô integral with their applications to modelling in science and engineering is very important offers. Also it is a comprehensive examination to the most important issues of stochastic differential equations. Due to computational complexity, in this study, we develop a new matrix approach based on 2D-OBP to solve two dimensional stochastic Volterra-Fredholm integral equations with the given boundary conditions. Based on this, 2-D operational matrix of integration and 2-D stochastic operational matrix of inte- 

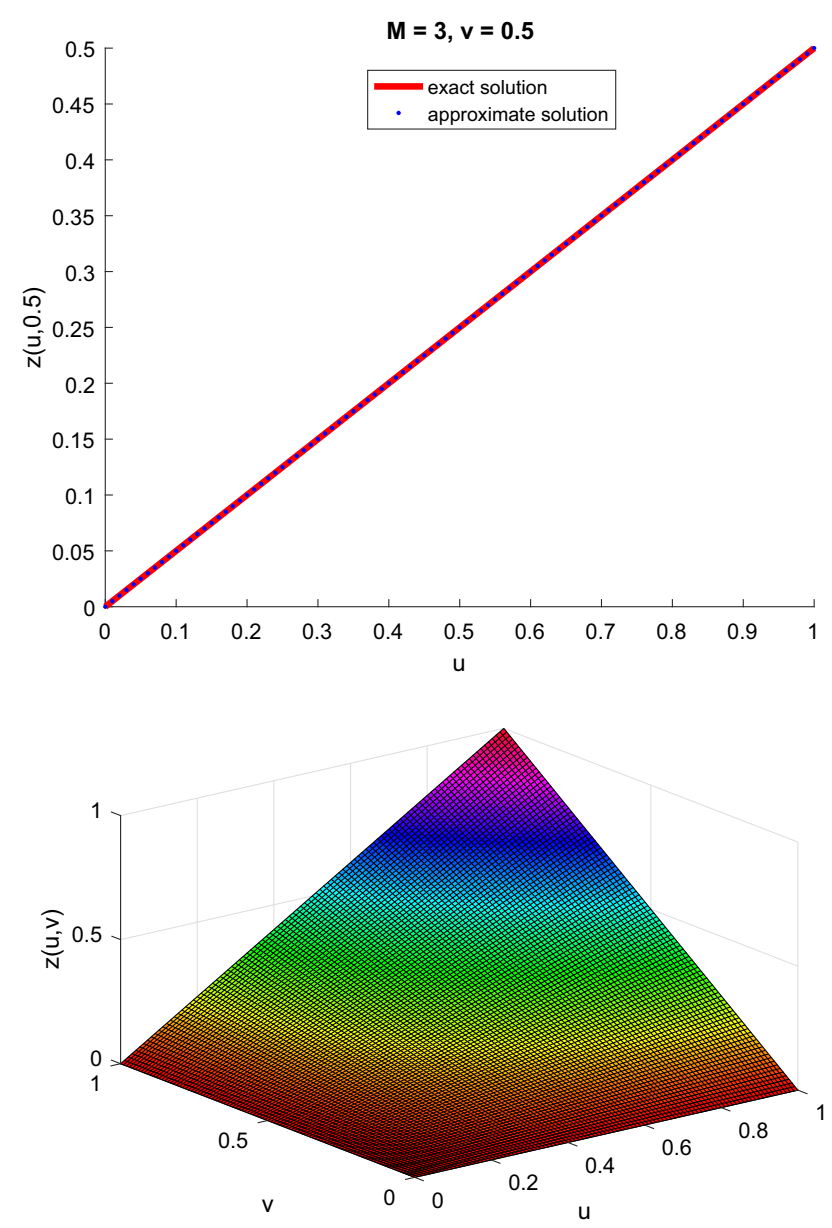

Fig. 10 The approximate solution and the exact solution for $v=0.5$ and $m=3$ and 3-D approximate solution of Example 5.3

gration is obtained, which leads to the converting the problem into a linear system. Some results and convergence analysis are also presented. Finally, by presenting three examples and reviewing the results and numerical comparisons, we showed that the proposed method has an excellent performance. In other words, numerical examples show that the suggested method is very accurate, simple and powerful tool to solve various problems. Another considerable advantage of this method in case of linear problems is that the exact solution can be obtained from $m$ th-order approximation when the solution is a polynomial of degree equal to or less than $m$.

Acknowledgements The authors are thankful to the respected reviewers for their valuable comments and constructive suggestions towards the improvement of the original paper.

Author Contributions Conceptualization, KS; Formal analysis, KS, AP and IM; Investigation, AP, SK and IM; Methodology, KS, AP and IM; Validation, SK and IM; Visualization, KS; Writing-original draft, KS, AP and IM All authors have read and agreed to the published version of the manuscript. 
Funding This research received no external funding.

Data availability statement Ph.D. thesis dissertations are public documents, but at the moment are not internetaccessible. If one is interested in revising the investigation, information can be requested from the authors.

\section{Declarations}

Conflict of interest The authors declare that there is no conflict of interest regarding the publication of this article.

\section{References}

1. Babaei, A., Jafari, H., Banihashemi, S., Ahmadi, M.: Mathematical analysis of a stochastic model for spread of Coronavirus. Chaos Solit. Fract. 145, 110788 (2021)

2. Ganji, R.M., Jafari, H., Moshokoa, S.P., Nkomo, N.S.: A mathematical model and numerical solution for brain tumor derived using fractional operator. Results Phys. 28, 104671 (2021)

3. Ganji, R.M., Jafari, H., Kgarose, M., Mohammadi, A.: Numerical solutions of time-fractional KleinGordon equations by clique polynomials. Alex. Eng. J. 60(5), 4563-4571 (2021)

4. Jafari, H., Ganji, R.M., Nkomo, N.S., Lv, Y.P.: A numerical study of fractional order population dynamics model. Results Phys. 104456 (2021)

5. Oguztoreli, M.N.: Time-Lag Control Systems. Academic Press, New York (1966)

6. Khodabin, M., Maleknejad, K., Rostami, M., Nouri, M.: Interpolation solution in generalized stochastic exponential population growth model. Appl. Math. Model. 36, 1023-1033 (2012)

7. Mode, C.J., Durrett, R., Klebaner, F., Olofsson, P.: Applications of stochastic processes in biology and medicine. Int. J. Stoch. Anal. (2013) (Article ID 790625, 2 pp). https://doi.org/10.1155/2013/790625

8. Oksendal, B.: Stochastic Differential Equations: An Introduction with Applications, 5th edn. SpringerVerlag, New York (1998)

9. Mohammadi, F.: Efficient Galerkin solution of stochastic fractional differential equations using second kind Chebyshev wavelets. Boletim da Sociedade Paranaense de Mathematica 35(1), 195-215 (2015)

10. Banihashemi, S., Jafari, H., Babaei, A.: A novel collocation approach to solve a nonlinear stochastic differential equation of fractional order involving a constant delay. Disc. Contin. Dyn. Syst. S (2021). https://doi.org/10.3934/dcdss.2021025

11. Banihashemi, S., Jafari, H., Babaei, A.: A stable collocation approach to solve a neutral delay stochastic differential equation of fractional order. J. Comput. Appl. Math. 403, 113845 (2022)

12. Kamrani, M.: Numerical solution of stochastic fractional differential equations. Numer. Algo. 68(1), $81-93(2015)$

13. Mirzaee, F., Samadyar, N.: Numerical solution of two dimensional stochastic Volterra-Fredholm integral equations via operational matrix method based on hat functions. SeMA J. 1-15 (2020)

14. Fallahpour, M., Khodabin, M., Maleknejad, K.: Theoretical error analysis and validation in numerical solution of two-dimensional linear stochastic Volterra-Fredholm integral equation by applying the block pulse functions. Cogent Math. 4(1), 1296750 (2017)

15. Asgari, M.: Block pulse approximation of fractional stochastic integro-differential equation. Commun. Numer. Anal. 1-7 (2014), https://doi.org/10.5899/2014/cna-00212

16. Taheri, Z., Javadi, S., Babolian, E.: Numerical solution of stochastic fractional integro-differential equation by the spectral collocation method. Comput. Appl. Math. 321, 336-47 (2017)

17. Sayevand, K., Machado, J.T., Masti, I.: On dual Bernstein polynomials and stochastic fractional integrodifferential equations. Math. Methods Appl. Sci. 43(17), 9928-9947 (2020)

18. Morters, P., Peres, Y.: Brownian Motion, vol. 30. Cambridge University Press, Cambridge (2010)

19. Napoli, A.: Solutions of linear second order initial value problems by using Bernoulli polynomials. Appl. Numer. Math. 99, 109-120 (2016)

20. Higham, D.J.: An algorithmic introduction to numerical simulation of stochastic differential equations. SIAM Rev. 43(3), 525-546 (2001) 
21. Samadyar, N., Mirzaee, F.: Numerical scheme for solving singular fractional partial integro-differential equation via orthonormal Bernoulli polynomials. Int. J. Numer. Model. Electron. Netw. Dev. Fields 32(6), e2652 (2019). https://doi.org/10.1002/jnm.2652

22. Klebaner, F.C.: Introduction to Stochastic Calculus with Applications. Monash University, Imperial College Presss, Australia (2005)

Publisher's Note Springer Nature remains neutral with regard to jurisdictional claims in published maps and institutional affiliations. 